\title{
Underwater Acoustic Communication by a Single-Vector Sensor: Performance Comparison Using Three Different Algorithms
}

\author{
Long-xiang Guo, ${ }^{1,2,3}$ Xiao Han $\mathbb{D}^{\mathrm{D}},{ }^{1,2,3}$ Jing-wei Yin, ${ }^{1,2,3}$ and Xue-song Yu ${ }^{1,2,3}$ \\ ${ }^{1}$ Acoustic Science and Technology Laboratory, Harbin Engineering University, Harbin 150001, China \\ ${ }^{2}$ Key Laboratory of Marine Information Acquisition and Security Harbin Engineering University, \\ Ministry of Industry and Information Technology, Harbin 150001, China \\ ${ }^{3}$ College of Underwater Acoustic Engineering, Harbin Engineering University, Harbin 150001, China \\ Correspondence should be addressed to Xiao Han; hanxiao1322@hrbeu.edu.cn
}

Received 24 June 2018; Accepted 8 October 2018; Published 1 November 2018

Academic Editor: Chao Tao

Copyright (C) 2018 Long-xiang Guo et al. This is an open access article distributed under the Creative Commons Attribution License, which permits unrestricted use, distribution, and reproduction in any medium, provided the original work is properly cited.

\begin{abstract}
In November, 2014, the underwater acoustic (UWA) communication experiment by a single-vector sensor was conducted in shallow water environment. In this paper, three different algorithms are used to process the experimental data and their performance are compared in terms of equalized output signal to noise ratio (OSNR) and bit error rate (BER). The three algorithms are P-DFE, B-DFE, and T-DFE, respectively. P-DFE uses only the pressure channel of the vector sensor to realize the decision feedback equalizer (DFE). B-DFE linearly combines the pressure channel and velocity channel first and then uses DFE to equalize the combined signal. T-DFE adopts time reversal to combine all the channels of the vector sensor and then is followed by a single-channel DFE to remove residual intersymbol interference (ISI). According to the data processing results, both B-DFE and T-DFE can achieve better performance compared with P-DFE. This paper also finds that the performance of B-DFE depends on the beam pattern of the combined signal while the performance of T-DFE depends on the $q$ function of the combined signal. Which algorithm should be used to process real data, B-DFE or T-DFE, depends on the degree of coherence between different channels of the vector sensor.
\end{abstract}

\section{Introduction}

UWA channels are characterized by large multipath spreads, rapidly time varying both in time and frequency domains, limited bandwidth, strong noise interference, and severe Doppler spreads which make them to be one of the most challenging environments in which to achieve robust communication [1]. In order to deal with the significant multipath and fast channel fluctuations, many approaches have been put forward among which the most commonly used are time reversal (TR) [2] and multichannel decision feedback equalizer (M-DFE) [3].

TR or phase conjugation is a physics-based approach to suppress multipath, and it can be generally divided into active TR and passive TR. TR, either active or passive, exploits spatial diversity to achieve spatial and temporal focusing in rich multipath environments. Temporal focusing (pulse compression) mitigates ISI. M-DFE is a signal processing-based approach to suppress multipath, and it can adaptively adjust the coefficients of the feedforward filter and feedback filter based on a different optimization criterion such as least mean squares (LMS) and recursive least squares (RLS) to dynamically match the CIR. Besides, M-DFE is usually embedded with a second-order phase lock loop (PLL) to tack the phase changes induced by channels. More details about TR and M-DFE can be found in $[4,5]$ and $[6,7]$, respectively. TR or M-DFE, a vertical array with a lot of spatially separated hydrophones, is usually used to achieve acceptable communication performance. But in practical operation, it is almost impossible to deploy a large aperture array to underwater platforms such as AUVs. Moreover, smaller array size is always preferred in underwater missions because it is easier to operate. 
Although UWA communication has been intensively investigated in the past years, existing works mainly focus on pressure sensor array, and only very limited research on the vector sensors is available. Acoustic vector sensors are capable of measuring three orthogonal particle velocity channels of the acoustic field, in addition to the scalar acoustic pressure, at a collocated point in space [8]. The waveforms of the pressure channel and velocity channel are demonstrated to have the same phase and totally coherent in theory provided that the source is in the far field [9], so one can do beamforming for vector sensor signals to suppress isotropic noise interference and improve the SNR of the received signal. Since the end of the last decade, the vector sensor has been widely used in many aspects of underwater missions such as underwater targets detection, direction of arrival (DOA) estimation, and underwater noise measurement [10-12]. Recently, the vector sensor began to be used in UWA communication. In UWA communications, different channels of vector sensors are always regarded as independent channels, so some spatial diversity-based equalizers have been used in vector sensor communication. It has been proposed in [13] that a single vector can serve as a multichannel receiver for UWA communication, and experimental results show that a single vector sensor can provide similar gain compared with a fourelement pressure sensor array. The same concluding can also be found in [14] which verifies that the single vector sensor has the same performance with the pressure sensor array at long communication range.

In this paper, we will use different algorithms to process the experimental data and compare their performance in terms of OSNR and BER. The three algorithms are P-DFE, B-DFE, and T-DFE, respectively. P-DFE uses only the pressure channel to realize the decision feedback equalizer while B-DFE linearly combines the pressure channel and velocity channel first (beamforming) and then uses DFE to equalize the combined signal. T-DFE adopts TR to fuse all the channels of the vector sensor and then is followed by a singlechannel DFE to remove residual ISI. P-DFE is very easy for readers to understand, so it will not be described in this paper.

The paper is organized as follows. Three different algorithms for UWA communication by vector sensors are introduced in Section 2. Experimental setup and CIRs are presented in Section 3. Data processing results are provided in Section 4, and concluding remarks are given in Section 5.

\section{Different Algorithms for UWA Communication by Vector Sensors}

2.1. B-DFE by the Vector Sensor. In this section, we suppose a plane wave propagation condition; i.e., the pressure, and the velocity channel of the acoustic field satisfy the acoustic Ohm's law [9], and their waveforms are totally coherent. The ratio of pressure and velocity channel equals to acoustic impedance of the medium, namely, $\rho c$, where $\rho$ is the density of the medium and $c$ is the acoustic speed. Denote $s(t)=$ $\left[p(t) \quad v_{x}(t) \quad v_{y}(t) \quad v_{z}(t)\right]^{\mathrm{T}}$ as the different channels of the vector sensor, where $p(t)$ is the pressure channel and $v_{x}(t)$, $v_{y}(t)$, and $v_{z}(t)$ are three orthogonal velocity channels. In this paper, we consider a two-dimensional vector sensor, and then the received signals can be described as

$$
\left\{\begin{array}{l}
p(t)=x(t), \\
v_{x}(t)=\frac{1}{\rho c} x(t) \cos \theta, \\
v_{y}(t)=\frac{1}{\rho c} x(t) \sin \theta
\end{array}\right.
$$

where $\theta$ is the horizontal azimuth of the acoustic arrivals and $\theta \in[0,2 \pi]$. Dipole directivity of the vector sensors can be electronic rotated. When talking about signal-processing problems, acoustic impedance in Formula (1) can be neglected. Velocity channels of the vector sensor have wideband directivity in the shape of " 8 " which is independent of signal frequency. Furthermore, this directivity can be rotated when it linearly combines with the two velocity channels $v_{x}(t)$ and $v_{y}(t)$ together. In this paper, we donate $v_{\mathrm{c}}(t)$ and $v_{\mathrm{s}}(t)$ as

$$
\left\{\begin{array}{l}
v_{\mathrm{c}}(t)=v_{x}(t) \cos \varphi+v_{y}(t) \sin \varphi=x(t) \cos (\theta-\varphi), \\
v_{\mathrm{s}}(t)=v_{x}(t) \sin \varphi+v_{y}(t) \cos \varphi=x(t) \sin (\theta-\varphi),
\end{array}\right.
$$

where $\varphi$ is the guide bearing. The directivity of $v_{\mathrm{c}}(t)$ and $v_{s}(t)$ will change as the guide bearing $\varphi$ changes. Besides, when combining the pressure channel and velocity channels together, the vector sensor can have multiple directivities. When source bearing is 0 , Figure 1 shows the directivity of $p(t)+v_{c}(t)$ and $p(t)+v_{s}(t)$. It can be seen that the maximum value of $p(t)+v_{c}(t)$ points to the source bearing, while the zero value points to the opposite direction of the source. The directivity of $p(t)+v_{s}(t)$ can be got when the directivity of $p(t)+v_{c}(t)$ is rotated clockwise by ninety degree.

From the above analysis, one may find that in order to make the directivity of the vector sensor point to the source, we should know the source bearing. So source bearing estimation is very important in vector sensor signal processing. In this paper, acoustic intensity average (AIA) is adopted to estimate source bearing. Consider a more practical model, and the received signal of the vector sensor can be written as

$$
\left\{\begin{array}{l}
p(t)=x(t)+\eta_{\mathrm{p}}(t) \\
v_{x}(t)=x(t) \cos \theta+\eta_{v_{x}}(t), \\
v_{y}(t)=x(t) \sin \theta+\eta_{v_{y}}(t),
\end{array}\right.
$$

where $\eta_{\mathrm{p}}(t), \eta_{v_{x}}(t)$, and $\eta_{v_{y}}(t)$ are received noise interference, and they are independent with $x(t)$. According to Formula (3), the block diagram of AIA is shown in Figure 2.

The outputs of AIA can be written as

$$
\begin{aligned}
\overline{\mathbf{I}}_{x}(t)= & \bar{p}(t) \bar{v}_{x}(t)=\bar{x}_{2}(t) \cos \theta+\bar{\eta}_{\mathrm{p}}(t) \bar{\eta}_{v_{x}}(t) \\
& +\bar{\eta}_{\mathrm{p}}(t) \bar{x}(t) \cos \theta+\bar{\eta}_{v_{x}}(t) \bar{x}(t), \\
\overline{\mathbf{I}}_{y}(t)= & \bar{p}(t) \bar{v}_{y}(t)=\bar{x}_{2}(t) \sin \theta+\bar{\eta}_{\mathrm{p}}(t) \bar{\eta}_{v_{y}}(t) \\
& +\bar{\eta}_{\mathrm{p}}(t) \bar{x}(t) \sin \theta+\bar{\eta}_{v_{y}}(t) \bar{x}(t),
\end{aligned}
$$




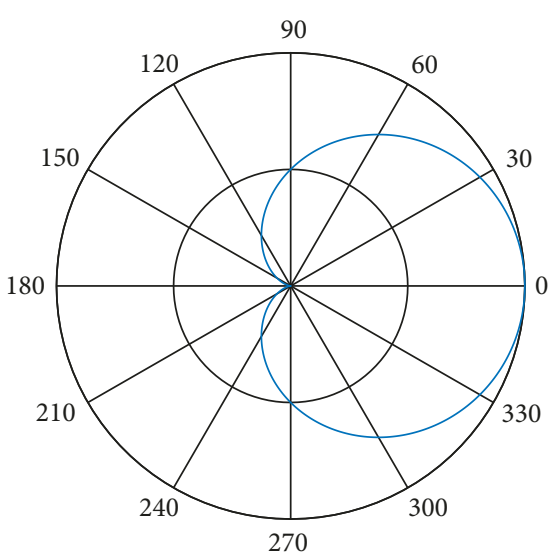

(a)

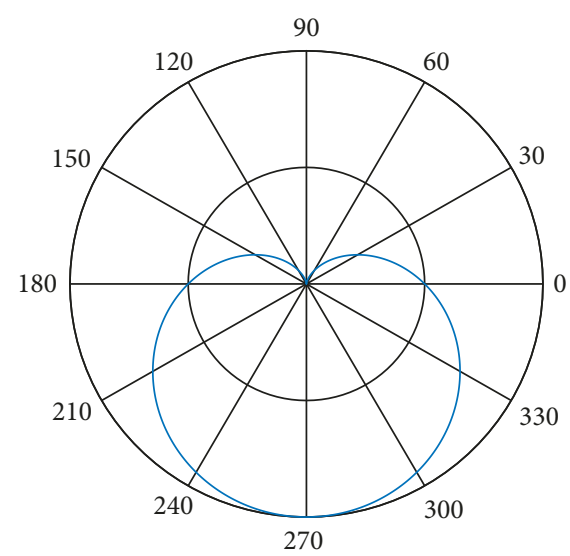

(b)

FIgURE 1: The directivity of the vector sensor when combining the pressure channel and velocity channels together: (a) $p(t)+v_{\mathrm{c}}(t)$; (b) $p(t)+v_{\mathrm{s}}(t)$. The source bearing is 0 .

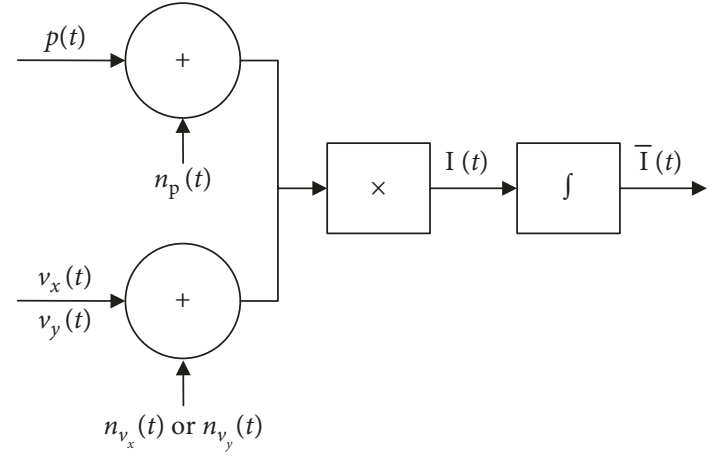

FIGURE 2: The block diagram of acoustic intensity average.

where the line above characters mean time average. As $\eta_{\mathrm{p}}(t)$, $\eta_{v_{x}}(t)$, and $\eta_{v_{y}}(t)$ are independent with $x(t)$, the last three terms of Formula (4) and Formula (5) can be regarded as small interference. So Formula (4) and Formula (5) can be rewritten as

$$
\begin{aligned}
& \overline{\mathbf{I}}_{x}(t)=\bar{p}(t) \bar{v}_{x}(t)=\bar{x}_{2}(t) \cos \theta+\Delta_{x}, \\
& \overline{\mathbf{I}}_{y}(t)=\bar{p}(t) \bar{v}_{y}(t)=\bar{x}_{2}(t) \sin \theta+\Delta_{y} .
\end{aligned}
$$

Then, we can estimate the source bearing by

$$
\bar{\theta} \triangleq \arctan \frac{\overline{\mathbf{I}}_{y}(t)}{\overline{\mathbf{I}}_{x}(t)}=\arctan \frac{\bar{p}(t) \bar{v}_{y}(t)}{\bar{p}(t) \bar{v}_{x}(t)} \text {. }
$$

Besides the source-bearing estimation, it will be necessary to discuss the processing gain of the linear combination (i.e., $\left.p+a \cdot v_{c}\right)$ of $p(t)$ and $v_{c}(t)$ here. In fact, researchers have done a lot of work on this. Suppose signal power and noise power in $p(t)$ are $\sigma_{s}^{2}$ and $\sigma_{\mathrm{n}}^{2}$, respectively. Signal power and noise power in $v_{\mathrm{c}}(t)$ will be $\sigma_{\mathrm{s}}^{2}$ and $\sigma_{\mathrm{n}}^{2} / 2$. So the SNR of $p+a \cdot v_{\mathrm{c}}$ can be described as

$$
\left(\frac{S}{N}\right)_{p+a v_{c}}=\frac{(1+a)^{2}}{\left(1+a^{2}\right) / 2} \cdot \frac{\sigma_{\mathrm{s}}^{2}}{\sigma_{\mathrm{n}}^{2}} .
$$

The SNR will reach a maximum value when $a=2$. Based on the above analysis, we can get the block diagram of B-DFE as shown in Figure 3. The data processing procedures may be generally divided into three steps: first, estimate the source bearing by AIA; second, linearly combine the pressure channel and velocity channels of the vector sensor; and third, DFE is then used to equalize the combined signal.

2.2. T-DFE by the Vector Sensor. The theory of TR has been widely introduced in many literatures, and we will not address again here. Figure 4 shows the block diagram of T-DFE by the vector sensor. Pressure channel and velocity channels are regarded as independent channels, and they are combined by TR. So the combined signal can be written as

$$
\begin{aligned}
r(n)= & p(n) * \widehat{h}_{\mathrm{p}}^{\dagger}(n,-l)+v_{x}(n) * \widehat{h}_{v_{x}}^{\dagger}(n,-l) \\
& +v_{y}(n) * \widehat{h}_{v_{y}}^{\dagger}(n,-l) \\
= & {\left[x(n) * h_{\mathrm{p}}(n, l)+\eta_{\mathrm{p}}(n)\right] * \widehat{h}_{\mathrm{p}}^{\dagger}(n,-l) } \\
& +\left[x(n) * h_{v_{x}}(n, l)+\eta_{v_{x}}(n)\right] * \widehat{h}_{v_{x}}^{\dagger}(n,-l) \\
& +\left[x(n) * h_{v_{y}}(n, l)+\eta_{v_{y}}(n)\right] * \widehat{h}_{v_{y}}^{\dagger}(n,-l) \\
= & x(n) *\left[h_{\mathrm{p}}(n, l) * \widehat{h}_{\mathrm{p}}^{\dagger}(n,-l)+h_{v_{x}}(n, l) * \widehat{h}_{v_{x}}^{\dagger}(n,-l)\right. \\
& \left.+h_{v_{y}}(n, l) * \widehat{h}_{v_{y}}^{\dagger}(n,-l)\right]+\eta_{\mathrm{p}}(n) * \widehat{h}_{\mathrm{p}}^{\dagger}(n,-l)+\eta_{v_{x}}(n) \\
& * \widehat{h}_{v_{x}}^{\dagger}(n,-l)+\eta_{v_{y}}(n) * \widehat{h}_{v_{y}}^{\dagger}(n,-l) \\
= & x(n) * q(n, l)+\Delta_{T} .
\end{aligned}
$$

In Equation (9), " $\dagger$ " denotes the conjugate operation and $0<l \leq L-1$, where $L$ is the discrete CIR length. The first term on the right side is the desired signal, and $q(n, l)$ can be 


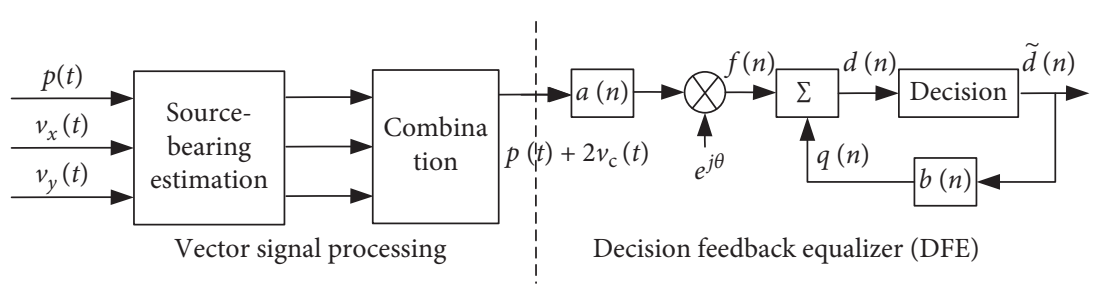

Figure 3: The block diagram of B-DFE.

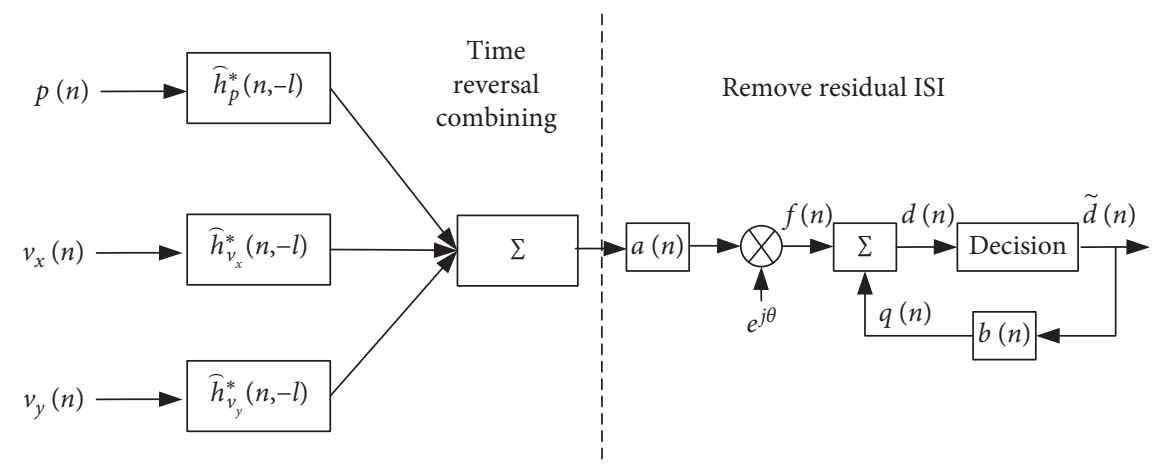

FIgURE 4: The block diagram of T-DFE.

regarded as the effective channel from the source to the vector sensor, which can be defined as

$$
\begin{aligned}
q(n, l)= & h_{\mathrm{p}}(n, l) * \widehat{h}_{\mathrm{p}}^{\dagger}(n,-l)+h_{v_{x}}(n, l) * \widehat{h}_{v_{x}}^{\dagger}(n,-l) \\
& +h_{v_{y}}(n, l) * \widehat{h}_{v_{y}}^{\dagger}(n,-l) .
\end{aligned}
$$

\section{Experimental Setup and CIRs}

The experiment was conducted at Songhua River with a water depth of about 7 meters in November 2014. Singlecarrier communication sequences are modulated using QPSK. The transmitted waveform is pulse shaped using a raised-cosine filter with a roll-off factor 0.7 . The center frequency is $12 \mathrm{kHz}$, the symbol rate is $2 \mathrm{k}$ symbols/s, and all signals are sampled at $96 \mathrm{kHz} .26000$ bits data were transmitted during this experiment, and the first 1000 bits were used as training $\left(N_{\mathrm{T}}=500\right)$. A transducer with the bandwidth from $8 \mathrm{kHz}$ to $20 \mathrm{kHz}$ was used as the source and deployed to $1.5 \mathrm{~m}$ water depth. A vector sensor, $1000 \mathrm{~m}$ away from the source, was deployed off the receiving boat which was always moored during this experiment. Note that the vector sensor was rigidly connected to the receiving boat through a steel pipe whose diameter was about $10 \mathrm{~cm}$.

During the experiment, the vector sensor was orderly deployed to two depths $(2.5 \mathrm{~m}$ and $1.5 \mathrm{~m})$, and the interval between adjacent depths is $1 \mathrm{~m}$. The same modulated signal was transmitted at every depth, so we can get two data sets denoted as "VS01" and "VS02." The measured CIRs for VS01 and VS02 are shown in Figures 5 and 6, respectively. One finds from these two figures that, the CIRS (no matter pressure channel or velocity channel) have very stable arrival structures over a time of $6 \mathrm{~s}$ for the two data sets. Besides, the structures of multipath are also very simple. There is only one dominant arrival for VS01 and two dominant arrivals for VS02. One can also notice from Figures 5(d) and 6(d) that the temporal coherence of CIRs is very high and channel coherence time is much longer than the packet length for these data.

\section{Experimental Results}

In this section, we will use three different algorithms, namely, P-DFE, B-DFE, and T-DFE, to process the experimental data. Figures 7 and 8 show the symbol-constellation plots for VS01 and VS02, respectively. In this paper, OSNR and BER are used as criterions to compare the performance of these three algorithms, and OSNR can be calculated by

$$
\text { OSNR }=10 \log \frac{\sum_{n=1}^{N}[x(n)-\bar{x}(n)]^{2}}{(1 / N) \sum_{n=1}^{N}[x(n)-\widetilde{d}(n)]^{2}},
$$

where $\bar{x}(n)$ is the average of the transmitted symbols and $\widetilde{d}(n)$ is the equalized symbols. The statistical OSNR and BER for the two data sets are shown in Table 1. According to the equalized symbol-constellation plots and statistical processing results, one can get the following conclusions: (1) acceptable performance can be achieved using only the pressure channels of the vector sensor in this experiment due to the relatively stable CIR and small multipath spread which is very easy to equalize for DFE; (2) both B-DFE and T-DFE outperform P-DFE which means that the vector sensor has better performance compared with the conventional pressure sensor; (3) compared with P-DFE, B-DFE achieves $1.26 \mathrm{~dB}$ and $1.79 \mathrm{~dB}$ gain, and T-DFE achieves $2.45 \mathrm{~dB}$ and $1.73 \mathrm{~dB}$ for VS01 and VS02, respectively.

According to Table 1, one can also find that when processing the experimental data by B-DFE, the gain for VS02 is higher than the gain for VS01. This result can be 


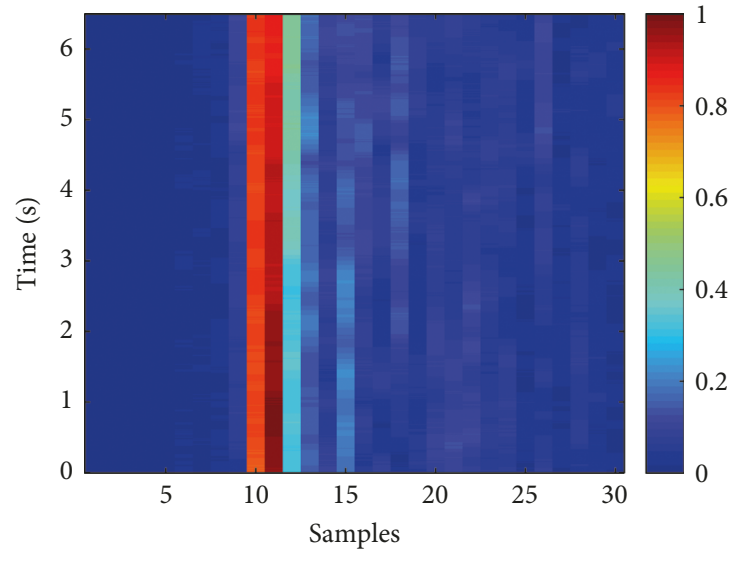

(a)

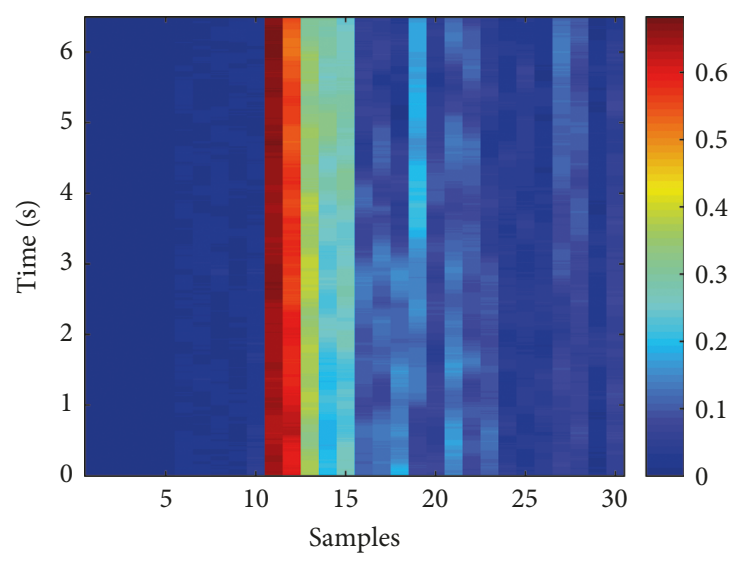

(c)

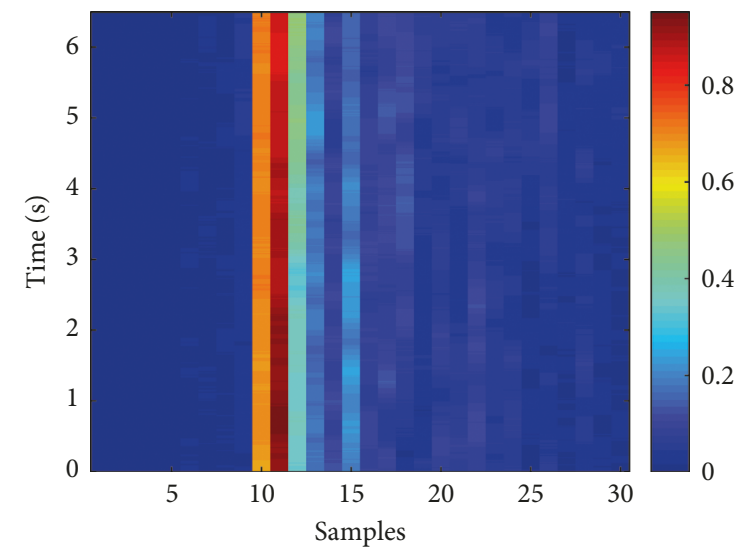

(b)

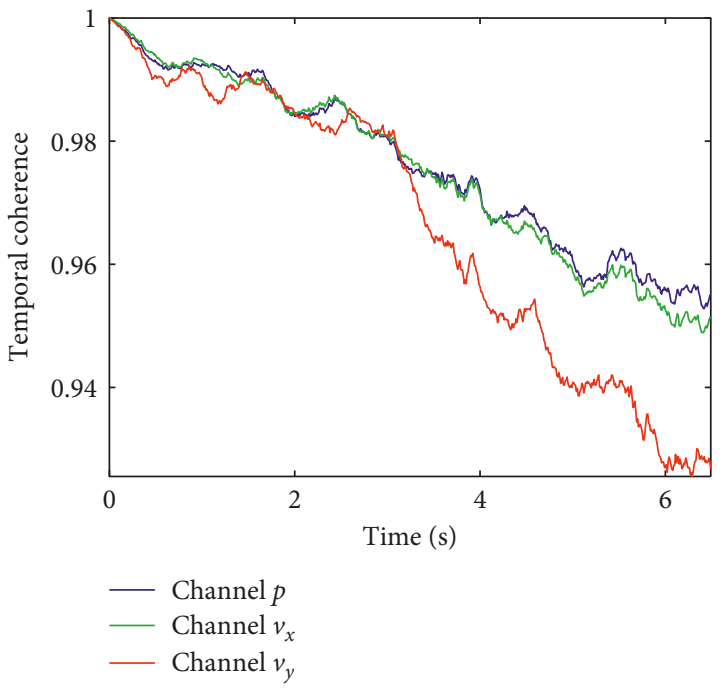

(d)

FIGURE 5: The measured CIRs for VS01: (a) the CIR for the pressure channel; (b) the CIR for the $v_{x}$ channel; (c) the CIR for the $v_{y}$ channel; (d) the temporal coherence of measured CIR.

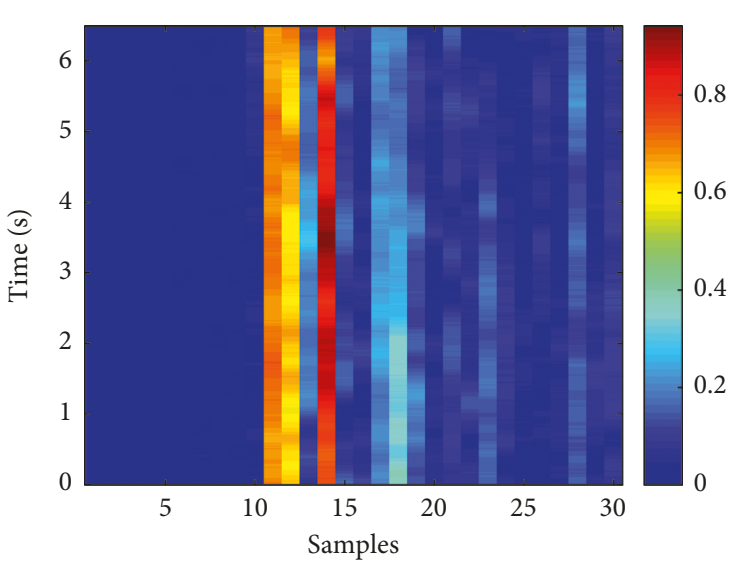

(a)

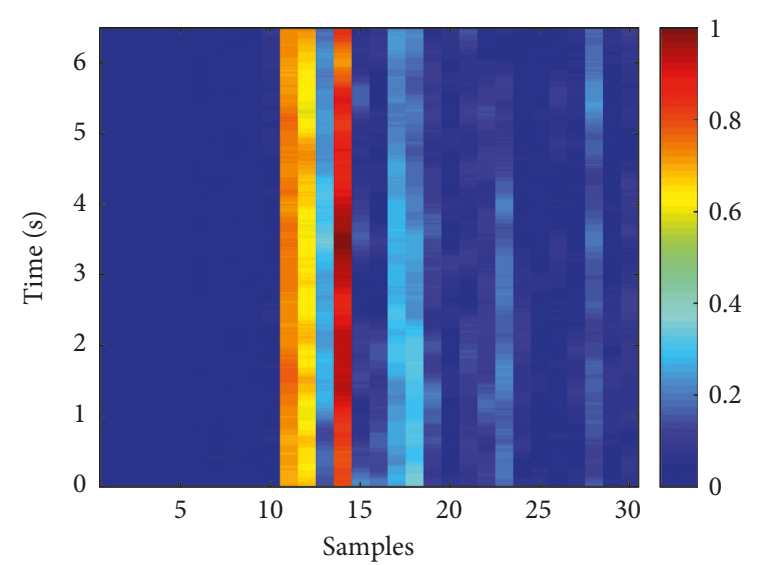

(b)

Figure 6: Continued. 

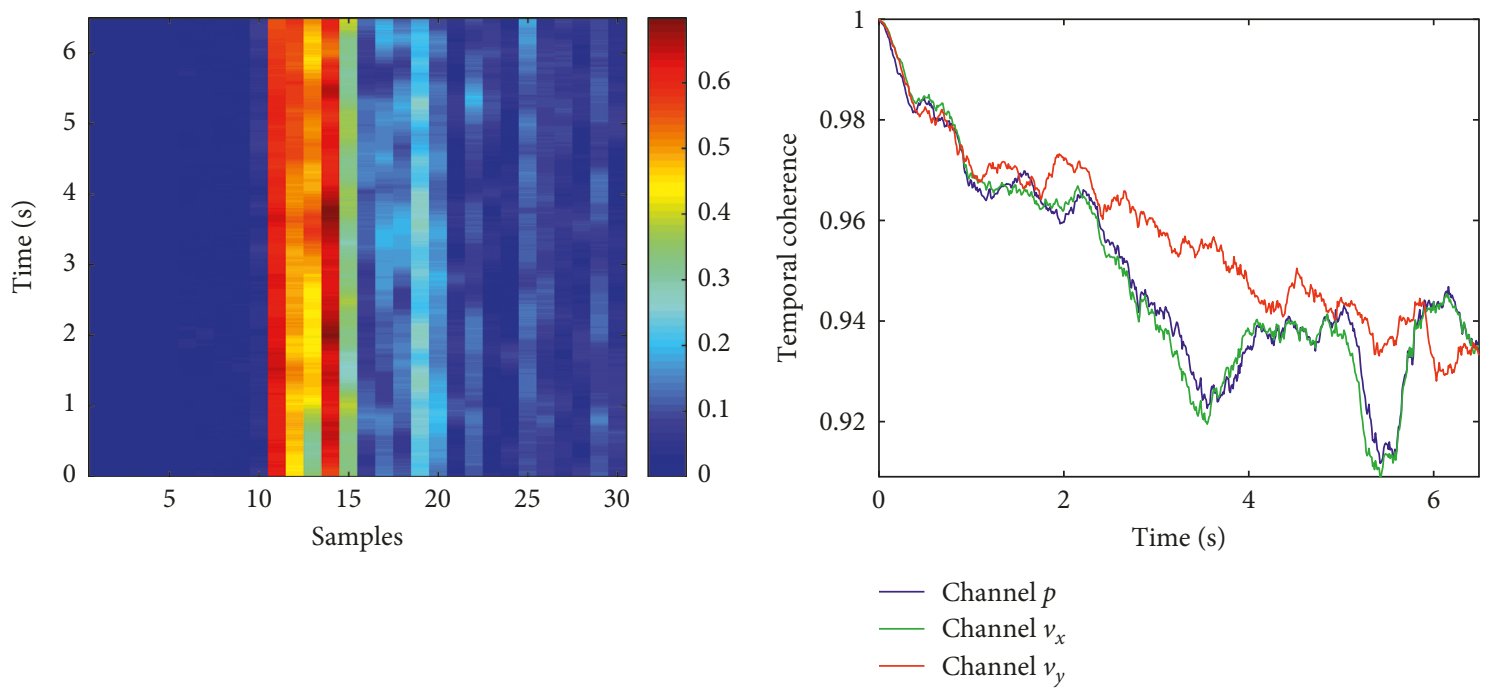

(c)

(d)

FIgURE 6: The measured CIRs for VS02: (a) the CIR for the pressure channel; (b) the CIR for the $v_{x}$ channel; (c) the CIR for the $v_{y}$ channel; (d) the temporal coherence of measured CIR.

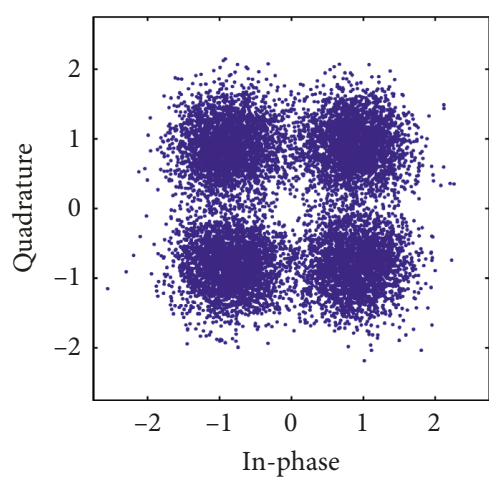

(a)

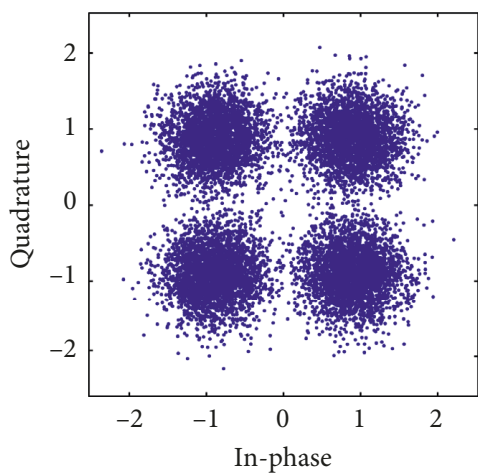

(b)

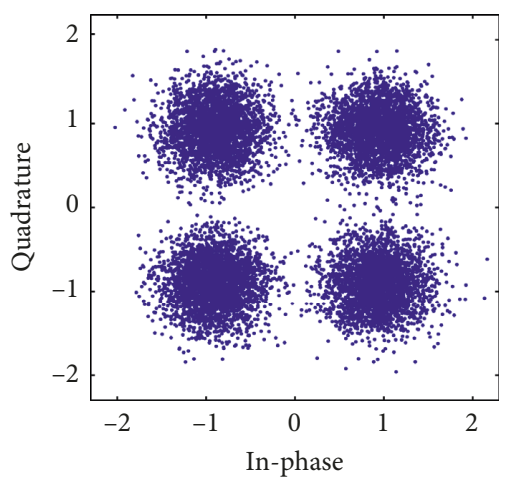

(c)

FIgURE 7: Symbol-constellation plots for VS01 using different algorithms: (a) P-DFE; (b) B-DFE; (c) T-DFE.

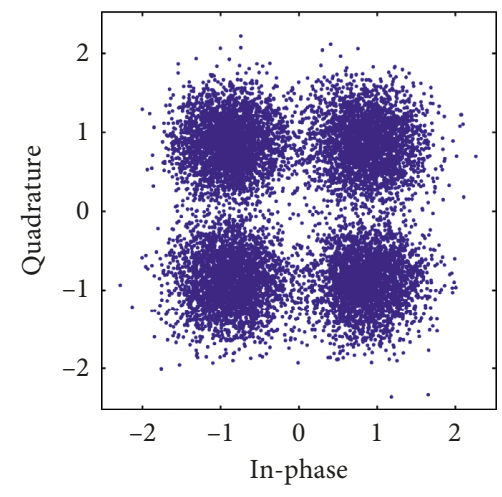

(a)

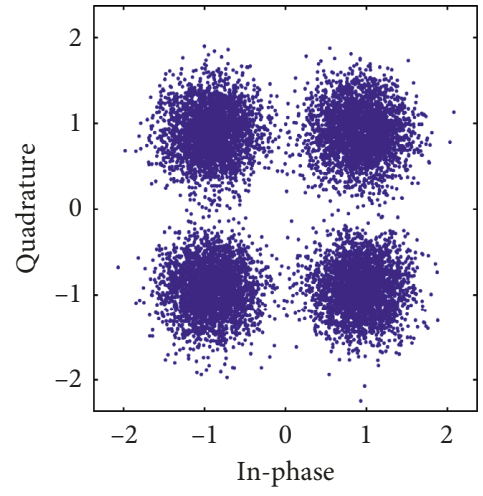

(b)

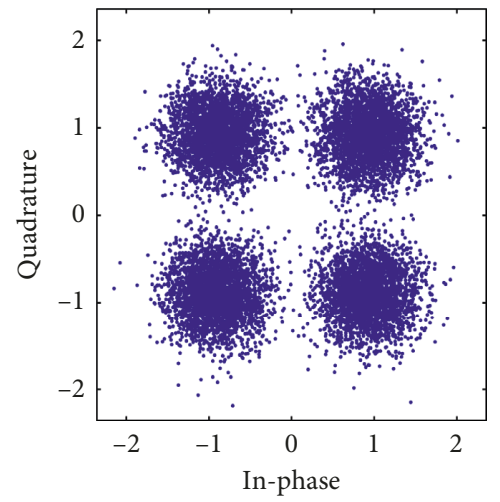

(c)

Figure 8: Symbol-constellation plots for VS02 using different algorithms: (a) P-DFE; (b) B-DFE; (c) T-DFE. 
TABLE 1: Statistical OSNR and BER for the two data sets.

\begin{tabular}{lcccc}
\hline & & VS01 & & VS02 \\
& OSNR (dB) & OSNR (dB) & BER (\%) \\
\hline P-DFE & 8.1 & 1.03 & 8.55 & 0.77 \\
B-DFE & 9.36 & 0.38 & 10.34 & 0.12 \\
T-DFE & 10.55 & 0.054 & 10.28 & 0.075 \\
\hline
\end{tabular}

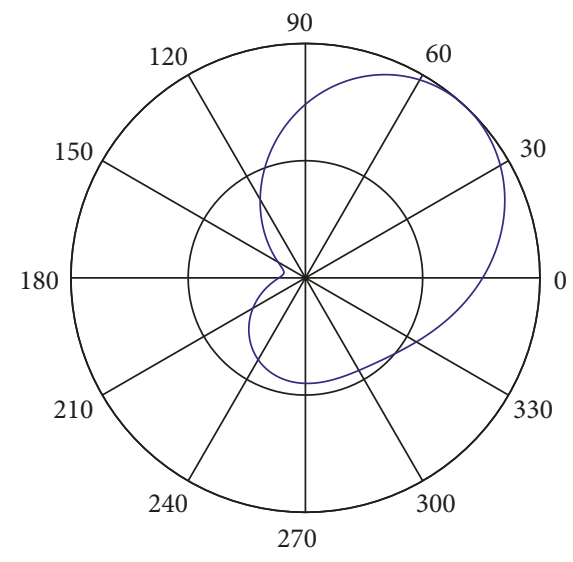

(a)

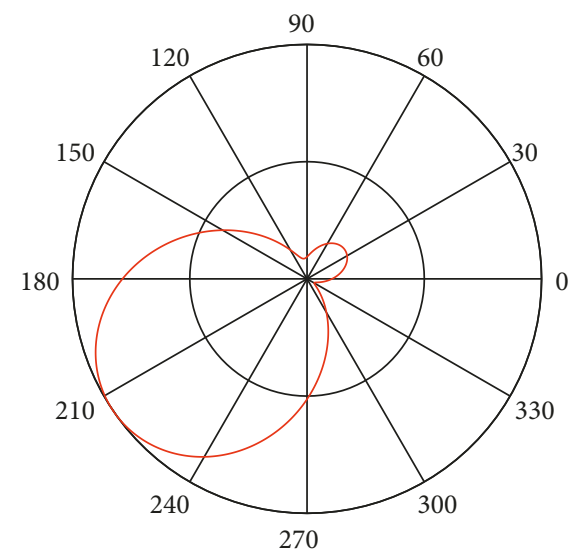

(b)

Figure 9: Beam patterns for experimental data sets: (a) VS01; (b) VS02.

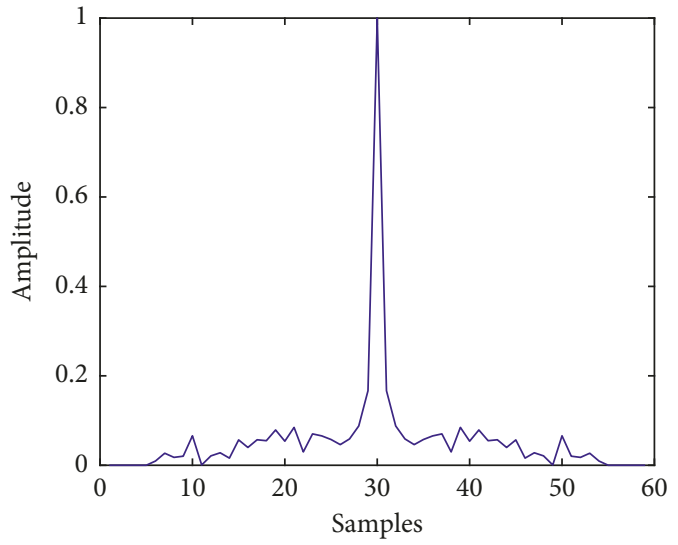

(a)

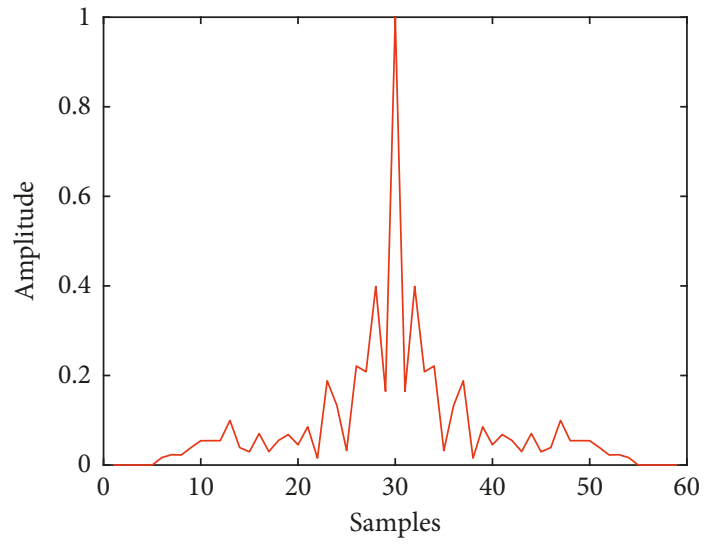

(b)

FIGURE 10: $q$ function for experimental data sets: (a) VS01; (b) VS02.

explained by beam patterns which are shown in Figure 9. The beam pattern for VS02 has narrower beam width which means that it has a better ability to suppress noise interference. The beam pattern for VS01 has wider beam width which means that it has a worse ability to suppress noise interference.

When processing the experimental data by T-DFE, one finds that the decoding result for VS01 is better than the decoding result for VS02 which is very different from the experiment results when processing by B-DFE. This phenomenon can be explained in terms of $q$ function of the vector sensor which is described by Formula (10). The $q$ functions estimated from experimental data are shown in Figure 10. It can be seen that the $q$ function for VS01 has lower side lobes and the main-to-side lobe ratio is about $10.7 \mathrm{~dB}$, which means it has a better ability to suppress ISI. The $q$ function for VS02 has higher side lobes, and the mainto-side lobe ratio is only about $4.0 \mathrm{~dB}$, which means it has a weaker ability to suppress ISI. Usually, the quality of $q$ function depends on the degree of incoherence and the number of channels. For a vector sensor, if the channels of the vector sensor are incoherent, the quality of $q$ function will be good, so T-DFE can achieve better performance but B-DFE will not perform well. Inversely, if the channels of the 
vector sensor are highly coherent, the beam pattern will be good, so B-DFE can achieve better performance, but T-DFE will not perform well. Besides, Table 1 shows that the OSNR of B-DFE is better than T-DFE, but the BER is opposed in VS02. These results from the OSNR are calculated by using the equalized symbols. In the B-DFE processing method, the constellation map is more aggregated, but there are multiple symbol decoding errors. And in the T-DFE processing method, the constellation diagram is relatively divergent, but there are fewer symbol decoding errors. Normally, the communication system has high OSNR with small BER.

\section{Conclusions}

Three different algorithms are adopted to process experimental data in this paper, and their performances are presented in terms of OSNR and BER. According to the data processing results, vector sensors always outperform the conventional pressure sensor which suggests that a smallscale vector sensor array can be used as an alternative to the pressure sensor array with large aperture for some compact underwater platforms such as AUVs. B-DFE and T-DFE both can achieve good performance in vector sensor signal processing. But which one can achieve the best performance, B-DFE or T-DFE, depends on the channel characteristics of the received signal.

\section{Data Availability}

The data used to support the findings of this study are available from the corresponding author upon request.

\section{Conflicts of Interest}

The authors declare that they have no conflicts of interest.

\section{Acknowledgments}

This research was supported by the National Natural Science Foundation of China (Grant no. 61631008, 61471137, and 50509059) and the Fundamental Research Funds for the Central Universities (Grant no. HEUCFM180503).

\section{References}

[1] D. B. Kilfoyle and A. B. Baggeroer, "The state of the art in under-water acoustic telemetry," IEEE Journal of Oceanic Engineering, vol. 25, no. 1, pp. 4-27, 2000.

[2] D. Rouseff, D. R. Jackson, W. L. J. Fox, C. D. Jones, J. A. Ritcey, and D. R. Dowling, "Underwater acoustic communication by passive-phase conjugation: theory and experimental results," IEEE Journal of Oceanic Engineering, vol. 26, no. 4, pp. 821831, 2001.

[3] M. Stojanovic, J. Catipovic, and J. G. Proakis, "Adaptive multichannel combining and equalization for underwater acoustic communications," Journal of the Acoustical Society of America, vol. 94, no. 3, pp. 1621-1631, 1993.

[4] J. A. Flynn, J. A. Ritcey, D. Rouseff, and W. L. J. Fox, "Multichannel equalization by decision-directed passive phase conjugation: experimental results," IEEE Journal of Oceanic Engineering, vol. 29, no. 3, pp. 824-836, 2004.
[5] T. C. Yang, "Correlation-based decision-feedback equalizer for underwater acoustic communications," IEEE Journal of Oceanic Engineering, vol. 30, no. 4, pp. 865-880, 2005.

[6] M. Pajovic and J. C. Preisig, "Performance analysis and optimal design of multichannel equalizer for underwater acoustic communications," IEEE Journal of Oceanic Engineering, vol. 40, no. 4, pp. 759-774, 2015.

[7] G. Zhang and H. Dong, "Joint passive-phase conjugation with adaptive multichannel combining for coherent underwater acoustic communications," Applied Acoustics, vol. 73, no. 4, pp. 433-439, 2012.

[8] A. Nehorai and E. Paldi, "Acoustic vector-sensor array processing," IEEE Transactions on Signal Processing, vol. 42, no. 9, pp. 2481-2491, 1994.

[9] S. Z. Liu, F. Zhou, Z. X. Sun, H. Li, and G. Qiao, "Experimental study of OFDM underwater acoustic communication using a vector hydrophone," Journal of Harbin Engineering University, vol. 33, no. 8, pp. 941-947, 2012.

[10] K. T. Wong and M. D. Zoltowski, "Closed-form underwater acoustic direction-finding with arbitrarily spaced vector hydrophones at unknown locations," IEEE Journal of Oceanic Engineering, vol. 22, no. 3, pp. 649-658, 1997.

[11] M. Hawkes and A. Nehorai, "Acoustic vector-sensor beamforming and Capon direction estimation," IEEE Transactions on Signal Processing, vol. 46, no. 9, pp. 2291-2304, 1998.

[12] M. Hawkes and A. Nehorai, "Acoustic vector-sensor correlations in ambient noise," IEEE Journal of Oceanic Engineering, vol. 26, no. 3, pp. 337-347, 2001.

[13] A. Ali and H. Guo, "A new compact multichannel receiver for underwater wireless communication networks," IEEE Transactions on Wireless Communications, vol. 8, no. 7, pp. 3326-3329, 2009.

[14] A. Song, A. Ali, M. Badiey, and H. Paul, "Experimental demonstration of underwater acoustic communication by vector sensors," IEEE Journal of Oceanic Engineering, vol. 36, no. 3, pp. 454-461, 2011. 


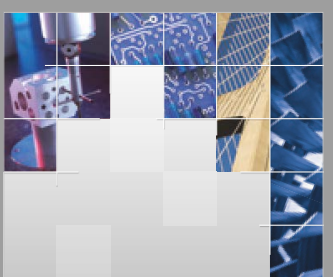

\section{Enfincering}
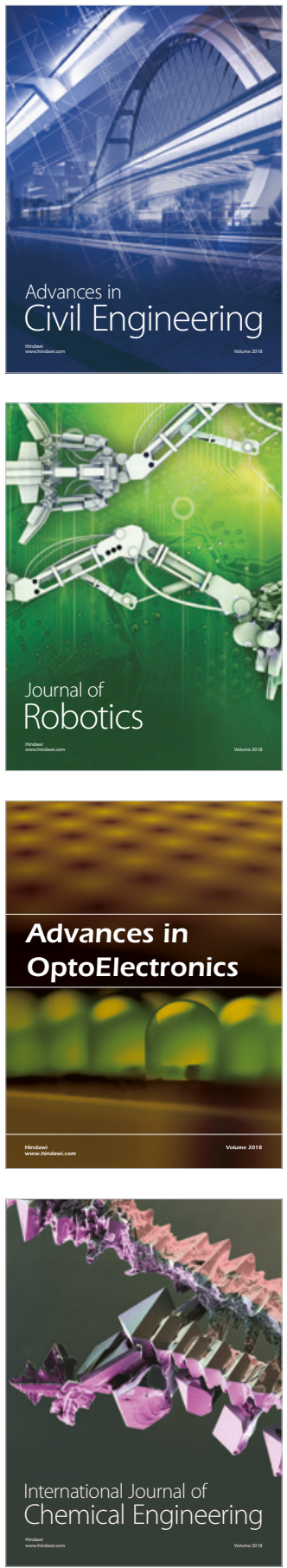

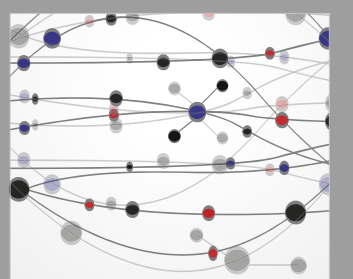

\section{Rotating \\ Machinery}

The Scientific World Journal

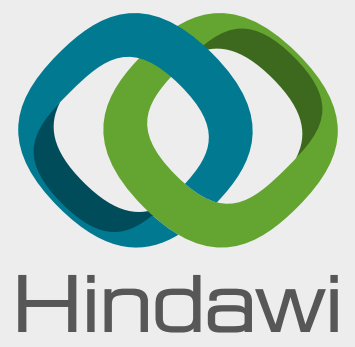

Submit your manuscripts at

www.hindawi.com
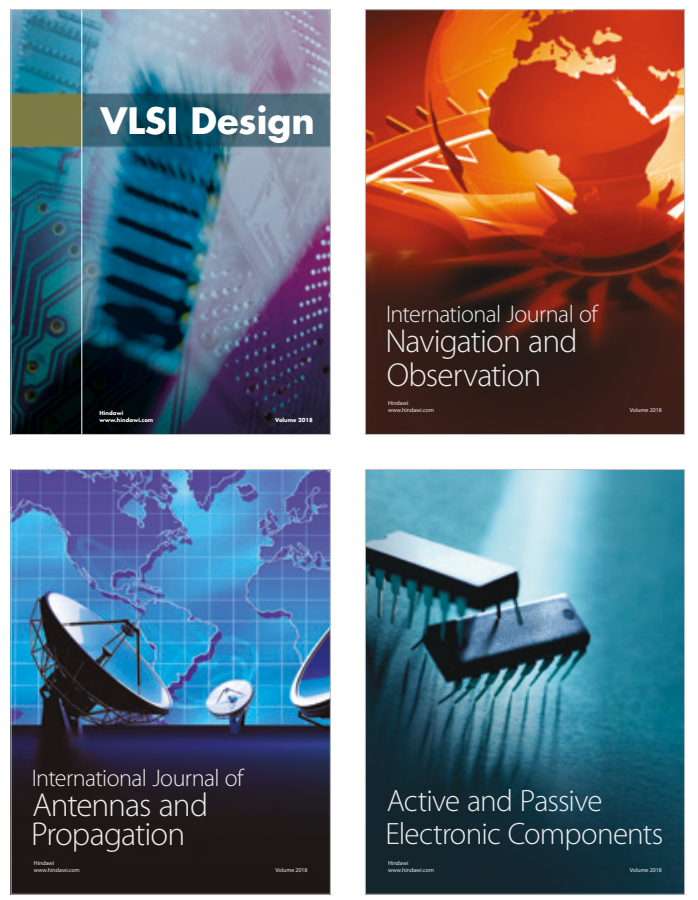
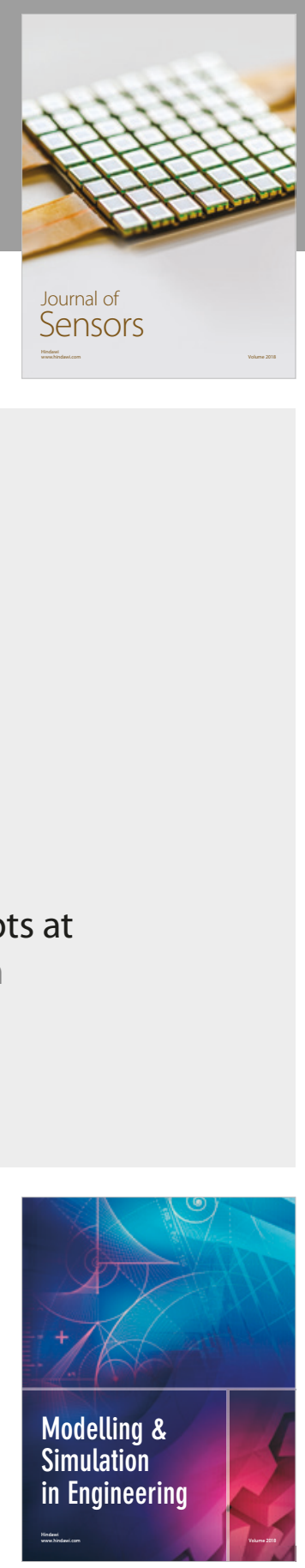

\section{Advances \\ Multimedia}
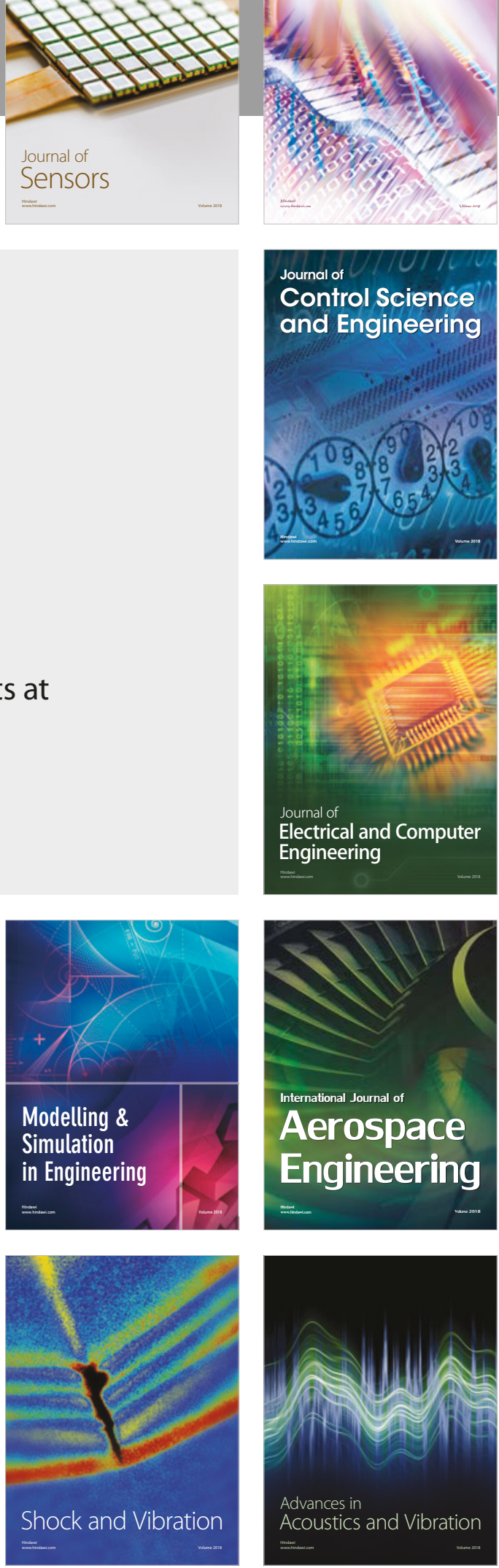